\title{
EARS - A WHO Platform for AI-Supported Real-Time Online Social Listening of COVID-19 Conversations
}

\author{
Tina D PURNAT ${ }^{\mathrm{a}}$, Harry WILSON ${ }^{\mathrm{b}}$, Tim NGUYEN ${ }^{\mathrm{c}, 1}$ and Sylvie BRIAND \\ ${ }^{a}$ Department of Digital Health and Innovation, Science Division, World Health \\ Organization, Geneva, Switzerland \\ ${ }^{\mathrm{b}}$ Citibeats, Barcelona, Spain \\ ${ }^{c}$ Department of Global Infectious Hazards Preparedness, Emergency \\ Preparedness, World Health Organization, Geneva, Switzerland
}

\begin{abstract}
As the COVID-19 pandemic evolves, the accompanying infodemic is being amplified through social media and has challenged effective response. The WHO Early AI-supported Response with Social Listening (EARS) is a platform that summarizes real-time information about how people are talking about COVID-19 in public spaces online in 20 pilot countries and in four languages. The aim of the platform is to better integrate social listening with other data sources and analyses that can inform infodemic response.
\end{abstract}

Keywords. infodemic, infodemic management, social listening, social media, pandemic preparedness, pandemic response

\section{Introduction}

Listening to people's questions and concerns is an important way for health authorities to learn about what matters to communities in response to COVID-19. WHO infodemic management framework [1] emphasizes the need for faster insights into concerns of citizens. The WHO EARS social listening platform [2] summarizes real-time information about how people are talking about COVID-19 in public spaces online, so risk communication and infodemic response interventions can be better designed and more effectively implemented.

\section{Methods}

The platform allows for exploration of the online public conversations at global and country level, a private country-specific dashboard for in-depth analysis, and an open API with the aggregated and anonymized data. The first version of the platform was launched in January 2021 covering 20 pilot countries from several WHO regions globally.

1 Corresponding Author, Tim Nguyen, Department of Global Infectious Hazards Preparedness, Emergency Preparedness, World Health Organization, Geneva, Switzerland; E-mail: nguyent@who.int. 
Data is collected daily from online COVID-19 conversations in English, Spanish, French and Portuguese from publicly available sources (ex Twitter, online forums, news comments, and blogs). Country attribution of the data is generated separately per data source. Each country sample consists of about 3,000 opinions per 1 million inhabitants per month (in total across all sources), except for small countries for which sampling ratio is higher. Data is then categorized automatically into 40 categories, with human quality controls, as per the WHO-developed COVID-19 public health social listening taxonomy. This is achieved by a semi-supervised machine learning algorithm with proprietary technology from Citibeats, an Ethical AI Platform. Full details on data sources, processing, aggregation and presentation are available in documentation section of the platform [2].

\section{Results}

Analysis results are presented normalized by the relative proportion of the conversation per country to ensure comparability across countries. The dashboards show how the topics of conversation change and evolve country by country over time, such as: what are the most popular categories and those gaining traction, and their patterns; what are the top and rising terms and hashtags within each category; what are the differences in conversations by; the composition of the conversation by intention: questions (confusion), complaints (frustration) or praise.

\section{Discussion}

After the launch of the platform in January 2021, the work will focus on evaluation and piloting of the platform with infodemic response teams in countries, and scaling it up to more countries and including more languages. More data sources be added to enable integrated infodemic landscape analysis, such as Google factchecker API, epidemiological data, and population surveys. Three research projects will be carried out to deep dive into development of new measurements and measure dimensions.

\section{Conclusions}

The EARS platform provides a public social listening tool from public online sources, that can be combined with other COVID-19 datasets for better integrated analysis to support infodemic response in countries.

\section{References}

[1] Tangcharoensathien V, Calleja N, Nguyen T, Purnat T, D’Agostino M, Garcia-Saiso S, Landry M, Rashidian A, Hamilton C, AbdAllah A, Ghiga I. Framework for managing the COVID-19 infodemic: methods and results of an online, crowdsourced WHO technical consultation. Journal of Medical Internet Research. 2020;22(6):e19659.

[2] WHO Early AI-supported Response with Social Listening platform. Available at: whoinfodemic.citibeats.com. Accessed 28 Jan 2021. 